\title{
SLC01B1 \& ApoE Gene Polymorphism Analysis of the Li People in Hainan Island and Its Clinical Significance
}

\author{
Heqiu Ruan ${ }^{1 *}$, Linlin Zhan ${ }^{2 *}$, Zihe Wang ${ }^{1}$, Mengjuan Xia ${ }^{1}$, Zhichao Ma ${ }^{1}$, Shengmiao Fu', \\ Xinping Chen 1 \\ ${ }^{1}$ Central Laboratory, Hainan General Hospital, Hainan Hospital Affiliated to the Hainan Medical College, Hainan \\ Provincial Key Laboratory of Cell and Molecular Genetic Translational Medicine, Haikou, China; ${ }^{2}$ Queen Mary \\ College, Nanchang University, Nanchang, China
}

Correspondence to: Xinping Chen, chenxinping52@126.com

Keywords: The Li People in Hainan Island, SLCO1B1, ApoE, Gene Polymorphisms, Statin Medicine

Received: February 4, $2021 \quad$ Accepted: March 26, $2021 \quad$ Published: March 29, 2021

Copyright $\odot 2021$ by author(s) and Scientific Research Publishing Inc.

This work is licensed under the Creative Commons Attribution International License (CC BY 4.0).

http://creativecommons.org/licenses/by/4.0/

\section{(c) (1) Open Access}

\section{ABSTRACT}

Objective: To analyze the distribution characteristics and clinical significance of SLCO1B1 and ApoE gene polymorphisms of the Li people in Hainan Island. Method: Selecting 502 high school students of the Li people from five cities and counties in Hainan Island (namely, Qiongzhong County, Dongfang City, Ledong County, Baoting County and Wuzhishan City) as research subjects in September, 2019; Applying PCR-fluorescence probe method to detect SLCO1B1 and ApoE genotypes of the Li people in Hainan Island, and statistically analyzing the distribution characteristics of gene frequency and the distribution differences in gene polymorphisms between different genders. Meanwhile, detecting the SLCO1B1 and ApoE gene of 527 people from the Han people in five regions mentioned before, so as to analyze the distribution differences of the SLCO1B1 and ApoE gene between the Han people and the Li people. Results: The frequency of each genotype of SLCO1B1 in the Li people in Hainan Island is: ${ }^{*} 1 \mathrm{a} /{ }^{*} 1 \mathrm{a} \quad 6.77 \%,{ }^{*} 1 \mathrm{a} /{ }^{*} 1 \mathrm{~b} \quad 27.09 \%,{ }^{*} 1 \mathrm{~b} / 1 \mathrm{~b} \quad 41.63 \%,{ }^{*} 1 \mathrm{a} /{ }^{*} 50.00 \%,{ }^{*} 1 \mathrm{a} /{ }^{*} 154.78 \%$, ${ }^{*} 1 \mathrm{~b} / 1516.93 \%$., ${ }^{*} 5 /{ }^{*} 50.00 \%,{ }^{*} /{ }^{*} 150.00 \%,{ }^{*} 15 /{ }^{*} 152.79 \%$; And that of ApoE is: e2/e2 $0.40 \%$, e2/e3 $17.73 \%$, e2/e4 $2.39 \%$, e3/e3 $65.54 \%$, e3/e4 $12.55 \%$, e4/e4 $1.39 \%$. There is no significant difference $(\mathrm{P}>0.05)$ in other genotypes except weak metabolic genotypes $\left(* 5 /{ }^{\star} 5\right.$, ${ }^{*} 5 /{ }^{*} 15$ and $\left.{ }^{*} 15 /{ }^{*} 15\right)$ between the Han and the Li peoples. Conclusion: The gene frequency of SLCO1B1 weak metabolic genotype is dramatically higher in the Li people of Hainan Island than that of the Han people in both Hainan Island and Central and South China, but there is no significant difference in ApoE gene frequency among them. Therefore, clinicians should

${ }^{\star}$ These authors contribute equally to this work. 


\section{adjust the dosage of statins and select the types of lipid-lowering drugs according to the differences in patients' genotypes, and strengthen the management of patients with ApoE4 risk gene.}

\section{INTRODUCTION}

Currently, Statins, namely 3-hydroxy-3 methylglutaryl-coenzyme A (HMG-CoA) reductase inhibitors, are the most effective lipid-lowering drugs and have become the most effective drugs for the prevention and treatment of coronary heart disease. The mechanism of statins action is reducing the intracellular cholesterol synthesis through competitively inhibiting the endogenous cholesterol synthesis rate-limiting enzyme HMG-CoA reductase and blocking the intracellular hydroxy mevalonate metabolism pathway, thereby stimulating the increase of the amount and activity of LDL receptors which are on the surface of the cell membrane, so as to clear serum cholesterol. The synthesis of LDL-C and VLDL is reduced by increasing the amount of LDL receptors on the liver cell membrane and accelerating the clearance of LDL, IDL [1]. Although different statins have the same functional group-hydroxyglutarate in chemical structure, the inheritance and variation of drug genes vary among individuals, especially affecting the coding gene of OATP1B1, SLCO1B1 and the SNP of ApoE's coding gene in several aspects, such as their pharmacokinetics (absorption, binding to plasma proteins, metabolism, and solubility), interactions with other drugs, drug efficacy, and adverse effects and so on. These aspects will vary dramatically among different individuals [2]. Therefore, it is important to conduct SLCO1B1 and APOE genotype testing to suggest the risk of adverse effects of statin use and to prevent cardiovascular and cerebrovascular disease, which can assist the clinicians to provide individualized medications for patients.

In this study, we use PCR-fluorescence probe method to detect the gene polymorphisms of SLCO1B1 and ApoE genotypes in the Li people in Hainan Island. At the molecular level, we analyze the distribution of SLCO1B1 and ApoE gene polymorphisms and learn the distribution differences of genotypes in the Li people of Hainan Island, so as to provide precise treatment and individualized medication for patients as well as to provide data support for prevention of diseases such as coronary heart disease, cardiovascular and cerebrovascular diseases, and dementia in the Li people of Hainan Island. The research report is followed.

\section{MATERIALS AND METHOD}

\subsection{Materials}

502 high school students of the Li people from five cities and counties in Hainan Island (namely, Qiongzhong County, Dongfang City, Ledong County, Baoting County, Wuzhishan City) are selected as research subjects in September, 2019. All of them are Li ethnic subjects who have long lived in the Li ethnic region of Hainan Island and have direct blood relatives within three generations. The average age is 16 \pm 1.3 years, 294 males and 232 females.

Randomly selecting 527 students in Han people from the same period in the above five cities and counties as the control group, and the subjects are all Han Chinese from Hainan Island with direct blood relatives within three generations and no history of intermarriage. Mean age is also $16 \pm 3.3$ years, 260 males and 242 females.

All above study individuals have no blood relationship and no history of blood transfusion. They are fully informed of the pros and cons. After obtaining informed consent signed by the subjects, $5 \mathrm{ml}$ of peripheral venous blood will be collected from the subjects, and use EDTA-K2 for anticoagulation.

\subsection{Method}

\subsubsection{DNA Extraction}

Take $2 \mathrm{ml}$ of EDTA-K2 anticoagulated venous blood, and use Smart 32 nucleic acid extractor for 
DNA extraction. Referring to the nucleic acid extraction kit instructions and instrument operating procedures. The concentration range of the extracted DNA is 5 - $15 \mathrm{ng} / \mathrm{ul}$.

\subsubsection{Instruments and Reagents}

1) Smart 32 nucleic acid extraction instrument: purchased from Sun Yat-sen University Da'an Gene Co., Ltd. PCR instrument.

2) Hongshi SLAN-48P fluorescent quantitative PCR instrument: purchased from Shanghai Hongshi Medical Technology Co., Ltd.

3) The DNA extraction kit: produced by Guangzhou Heshi Biotechnology Co., Ltd.

4) SLCO1B1 and ApoE gene polymorphism detection kit (PCR-fluorescent probe method): produced by Wuhan Youzhiyou Medical Technology Co., Ltd.

\subsubsection{PCR Amplification}

According to the instructions of the SLCO1B1 and ApoE gene polymorphism detection reagents produced by Wuhan Youzhiyou Medical Technology Co., Ltd., the PCR amplification steps include: first, add the SLCO1B1*1b reaction solution, SLCO1B1*5 reaction solution, ApoE2 reaction solution, ApoE4 reaction solution sequentially into a $200 \mu \mathrm{L}$ PCR 8-strip tube, and each solution is $23 \mu \mathrm{L}$, then add $2 \mu \mathrm{L}$ each of genomic DNA, weak positive control solution, and blank control solution to the reaction tube which contains 4 kinds of PCR reaction solutions. Cover the reaction tube and start the amplification according to the following procedure: maintain a constant temperature of $37^{\circ} \mathrm{C}$ for 10 minutes; pre-denaturation at $95^{\circ} \mathrm{C}$ for 5 minutes, denaturation at $95^{\circ} \mathrm{C}$ for 15 seconds, extension at $60^{\circ} \mathrm{C}$ for 60 seconds, and amplification for 40 times.

\subsubsection{Results Interpretation}

1) Sample Results: The ROX channel of all the samples (endogenous reference gene): Ct value is lower than or equal to 32 , with an obvious amplification curve. When the Ct value of FAM channel is lower than 38 and the Ct value of VIC channel is greater than or equal to 38 or no Ct value, it is homozygous wild type (Figure 1(A)); when the Ct value of FAM channel is lower than 38 and the Ct value of VIC channel is lower than 38, it is heterozygous mutant type (Figure 1(B)); when the Ct Value of FAM channel is greater than or equal to 38 or there is no Ct value and the Ct value of VIC channel lower than 38 , it is a homozygous mutant (Figure 1(C)).

2) Quality Control: Weak positive quality control should be in FAM, VIC and ROX channels, and the Ct value is less than or equal to $\leq 32$; the amplification curve has an obvious exponential growth period. The blank control should have no amplification curve in FAM, VIC and ROX channels, or the Ct value is greater than or equal to 38 or there is no Ct value.

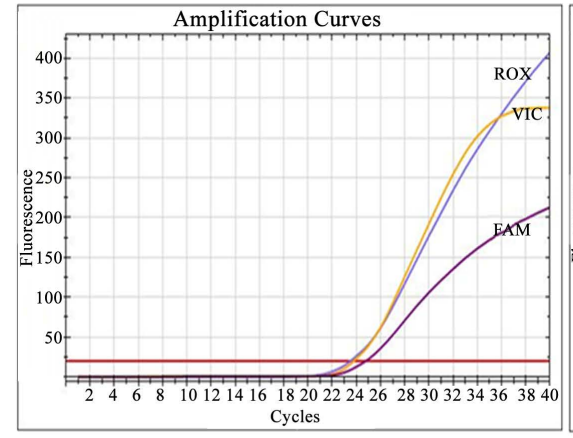

(A)

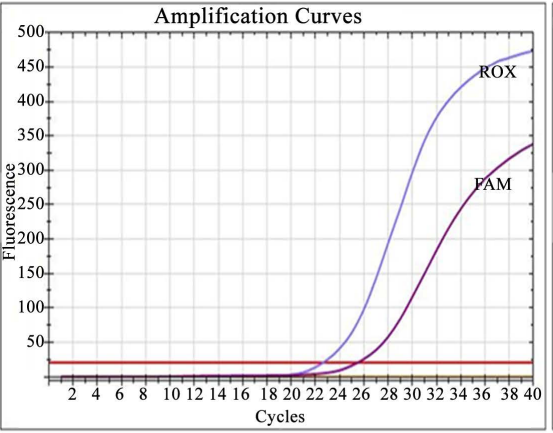

(B)

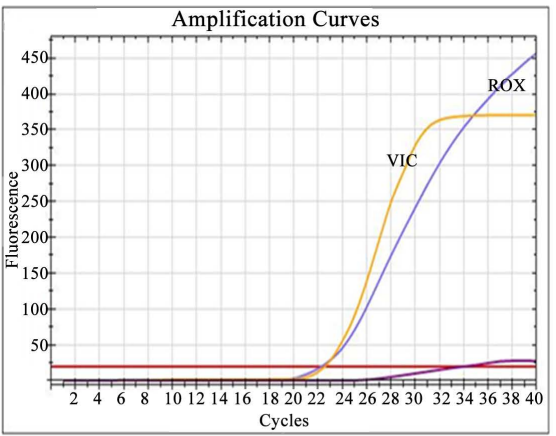

(C)

Figure 1. The amplification curve of SCLO1B1 and ApoE gene. Note: (A) Homozygous wild type; (B) Heterozygous mutant type; (C) Homozygous mutant. 


\subsection{Statistical Method}

The genotype frequency and allele frequency of each SNP locus are calculated by using frequency calculation method. The $\chi^{2}$ test is used to analyze whether each SNP locus conformed to the Hardy-Weinberg equilibrium, and the SPSS21.0 statistical software is used for statistical analysis. The count data was expressed as the number of cases. And the $\chi^{2}$ test is also used to compare genetic makeup ratios and alleles among different groups. $\mathrm{P}<0.05$ indicates that the difference reaches statistical significance.

\section{RESULTS}

\subsection{Analysis of the Genotype Distribution of SCLO1B1 and ApoE and the Frequency Distribution of Alleles between the Li People and the Han People in Hainan Island}

1) The data about the genotype distribution of SCLO1B1 and ApoE and the gene frequency of alleles between 502 subjects from the Li people in Hainan Island used in this study are presented in Table 1. The $\chi^{2}$ test indicates that the gene frequency of SLCO1B1 and ApoE is conformed to the Hardy-Weinberg equilibrium (SLCO1B1 388A $>$ G, 521T $>$ C and ApoE 388T $>C$, 526C $>$ T and P > 0.05), so the Li people selected in the study is representative.

2) The data about the genotype distribution of SCLO1B1 and ApoE and the frequency of alleles of 527 subjects from the Han people in Hainan Island (control group) used in this study are presented in Table 2.

The $\chi^{2}$ test indicates that the gene frequency of SLCO1B1 and ApoE is conformed to the Hardy-Weinberg equilibrium (SLCO1B1 388A $>$ G, 521T $>C$ and $A p o E 388 T>C$, 526C $>$ T and $\mathrm{P}>0.05$ ), so the Han people selected in the study is representative.

Table 1. Frequency distribution results of SLCO1B1 and ApoE gene polymorphisms in the Li people.

\begin{tabular}{|c|c|c|c|c|c|c|c|c|}
\hline \multirow{2}{*}{ Gene } & \multirow{2}{*}{$\begin{array}{c}\text { SNP } \\
(\mathrm{n}=502)\end{array}$} & \multirow{2}{*}{ Genotype } & \multicolumn{2}{|c|}{ Genotype } & \multirow{2}{*}{ Allele } & \multicolumn{2}{|c|}{ Allele } & \multirow{2}{*}{$\begin{array}{c}x^{2}, \\
\text { the value } \\
\text { of } \mathrm{P}\end{array}$} \\
\hline & & & Number (n) & Ratio (\%) & & Frequency & Ratio (\%) & \\
\hline \multirow{6}{*}{ SLCO1B1 } & \multirow{3}{*}{$\begin{array}{c}388 A>G \\
\text { rs2306283 }\end{array}$} & AA & 34 & $6.77 \%$ & $\mathrm{~A}$ & 228 & $22.71 \%$ & \\
\hline & & AG & 160 & $31.87 \%$ & G & 776 & $77.29 \%$ & $4.76>0.05$ \\
\hline & & GG & 308 & $61.36 \%$ & & & & \\
\hline & \multirow{3}{*}{$\begin{array}{c}521 \mathrm{~T}>\mathrm{C} \\
\mathrm{rs} 4149056\end{array}$} & $\mathrm{TT}$ & 379 & $75.50 \%$ & $\mathrm{~T}$ & 867 & $86.35 \%$ & \\
\hline & & $\mathrm{TC}$ & 109 & $21.71 \%$ & $\mathrm{C}$ & 137 & $13.65 \%$ & $3.13>0.05$ \\
\hline & & $\mathrm{CC}$ & 14 & $2.79 \%$ & & & & \\
\hline \multirow{6}{*}{ ApoE } & \multirow{3}{*}{$\begin{array}{c}388 \mathrm{~T}>\mathrm{C} \\
\mathrm{rs} 429358\end{array}$} & $\mathrm{TT}$ & 420 & $83.67 \%$ & $\mathrm{~T}$ & 915 & $91.14 \%$ & \\
\hline & & $\mathrm{TC}$ & 75 & $14.94 \%$ & $\mathrm{C}$ & 89 & $8.86 \%$ & $2.79>0.05$ \\
\hline & & $\mathrm{CC}$ & 7 & $1.39 \%$ & & & & \\
\hline & \multirow{3}{*}{$\begin{array}{c}526 \mathrm{C}>\mathrm{T} \\
\mathrm{rs} 7412\end{array}$} & $\mathrm{CC}$ & 399 & $79.48 \%$ & $\mathrm{C}$ & 899 & $89.54 \%$ & \\
\hline & & $\mathrm{CT}$ & 101 & $20.12 \%$ & $\mathrm{~T}$ & 105 & $10.46 \%$ & $2.75>0.05$ \\
\hline & & $\mathrm{TT}$ & 2 & $0.40 \%$ & & & & \\
\hline
\end{tabular}


Table 2. Frequency distribution results of SLCO1B1 and ApoE gene polymorphisms in the Han people.

\begin{tabular}{|c|c|c|c|c|c|c|c|c|}
\hline \multirow{2}{*}{ Gene } & \multirow{2}{*}{$\begin{array}{c}\text { SNP } \\
(\mathrm{n}=853)\end{array}$} & \multirow{2}{*}{ Genotype } & \multicolumn{2}{|c|}{ Genotype } & \multirow{2}{*}{ Allele } & \multicolumn{2}{|c|}{ Allele } & \multirow{2}{*}{$\begin{array}{c}\chi^{2} \text {, the } \\
\text { value of } P\end{array}$} \\
\hline & & & Number (n) & Ratio (\%) & & Frequency & Ratio (\%) & \\
\hline \multirow{6}{*}{ SLCO1B1 } & \multirow{3}{*}{$\begin{array}{c}388 \mathrm{~A}>\mathrm{G} \\
\mathrm{rs} 2306283\end{array}$} & AA & 23 & $4.36 \%$ & $\mathrm{~A}$ & 247 & $23.43 \%$ & \\
\hline & & AG & 201 & $38.14 \%$ & G & 807 & $76.57 \%$ & $2.09>0.05$ \\
\hline & & GG & 303 & $57.50 \%$ & & & & \\
\hline & \multirow{3}{*}{$\begin{array}{c}521 \mathrm{~T}>\mathrm{C} \\
\mathrm{rs} 4149056\end{array}$} & $\mathrm{TT}$ & 402 & $76.28 \%$ & $\mathrm{~T}$ & 922 & $87.48 \%$ & \\
\hline & & $\mathrm{TC}$ & 118 & $22.39 \%$ & $\mathrm{C}$ & 132 & $12.52 \%$ & $0.26>0.05$ \\
\hline & & $\mathrm{CC}$ & 7 & $1.33 \%$ & & & & \\
\hline \multirow{6}{*}{ ApoE } & \multirow{3}{*}{$\begin{array}{c}388 \mathrm{~T}>\mathrm{C} \\
\mathrm{rs} 429358\end{array}$} & TT & 453 & $85.96 \%$ & $\mathrm{~T}$ & 978 & $92.79 \%$ & \\
\hline & & $\mathrm{TC}$ & 72 & $13.66 \%$ & $\mathrm{C}$ & 76 & $7.21 \%$ & $0.23>0.05$ \\
\hline & & $\mathrm{CC}$ & 2 & $0.38 \%$ & & & & \\
\hline & \multirow{3}{*}{$\begin{array}{c}526 \mathrm{C}>\mathrm{T} \\
\mathrm{rs} 7412\end{array}$} & $\mathrm{CC}$ & 414 & $78.56 \%$ & $\mathrm{C}$ & 935 & $88.71 \%$ & \\
\hline & & CT & 107 & $20.30 \%$ & $\mathrm{~T}$ & 119 & $11.29 \%$ & $0.28>0.05$ \\
\hline & & $\mathrm{TT}$ & 6 & $1.14 \%$ & & & & \\
\hline
\end{tabular}

\subsection{Analysis on the Differences of the Distribution of SLC01B1 and ApoE Gene Polymorphisms} between the Li People and the Han People in Hainan Island

In this study, the polymorphisms of genetic locus SLCO1B1 ${ }^{\star} 1 \mathrm{~b} 388 \mathrm{~A}>\mathrm{G}$, SLCO1B1 ${ }^{\star} 5521 \mathrm{~T}>\mathrm{C}$ of SLCO1B1 and APOE2 526C >T, APOE4 388T $>$ C of APOE from $502 \mathrm{Li}$ people and 527 Han people in Hainan island are analyzed. In Hainan Island, the frequencies of each SLCO1B1 genotypes in the Li people are ${ }^{\star} 1 \mathrm{a} /{ }^{\star} 1 \mathrm{a} 6.77 \%,{ }^{\star} 1 \mathrm{a} /{ }^{*} 1 \mathrm{~b} 27.09 \%,{ }^{\star} 1 \mathrm{~b} / 1 \mathrm{~b} 41.63 \%,{ }^{*} 1 \mathrm{a} /{ }^{\star} 50.00 \%,{ }^{*} 1 \mathrm{a} /{ }^{\star} 154.78 \%,{ }^{*} 1 \mathrm{~b} / 1516.93 \%,{ }^{\star} 5 /{ }^{*} 50.00 \%$, ${ }^{\star} 5 /{ }^{\star} 150.00 \%,{ }^{\star} 15 /{ }^{\star} 152.79 \%$. And that of each ApoE genotypes in the Li people are e2/e2 $0.40 \%$, e2/e3 $17.73 \%$, e2/e4 2.39\%, e3/e3 65.54\%, e3/e4 12.55\%, e4/e4 1.39\% (Table 3). In the control group, the frequencies of SLCO1B1 genotypes in the Han people are ${ }^{\star} 1 \mathrm{a} /{ }^{\star} 1 \mathrm{a} 4.36 \%,{ }^{\star} 1 \mathrm{a} /{ }^{\star} 1 \mathrm{~b} 32.07 \%,{ }^{\star} 1 \mathrm{~b} / 1 \mathrm{~b} 39.8 \%$, ${ }^{\star} 1 \mathrm{a} /{ }^{\star} 50.00 \%,{ }^{\star} 1 \mathrm{a} /{ }^{\star} 156.07 \%,{ }^{\star} 1 \mathrm{~b} / 1516.32 \%,{ }^{\star} 5 /{ }^{\star} 50.00 \%,{ }^{*} 5 /{ }^{\star} 150.00 \%,{ }^{\star} 15 /{ }^{\star} 151.33 \%$. And that of ApoE genotypes in the Han people are e2/e2 1.14\%, e2/e3 18.22\%, e2/e4 2.09\%, e3/e3 66.60\%, e3/e4 $11.57 \%$, e4/e4 0.38\% (Table 4).

The $\chi^{2}$ test indicates that the frequencies of SLCO1B $1{ }^{\star} 15 /{ }^{\star} 15$ are significantly different between $\mathrm{Li}$ and Han peoples in Hainan Island $(\mathrm{P}<0.05)$, while the difference in frequencies of other SLCO1B1 genotypes and ApoE genotypes are not significantly different.

\subsection{Analysis on Differences of the Proportions of Three Metabolic Types of SLC01B1 Gene between Li People and Han People in Hainan Island}

According to the risk degree of rhabdomyolysis or myopathy caused by mutant SLCO1B1 gene and the dosage of drugs, SLCO1B1 gene is divided into three metabolic types: normal metabolic type $\left({ }^{*} 1 \mathrm{a} /{ }^{*} 1 \mathrm{a}\right.$, $\left.{ }^{\star} 1 \mathrm{a} /{ }^{\star} 1 \mathrm{~b},{ }^{\star} 1 \mathrm{~b} / 1 \mathrm{~b}\right)$, intermediate metabolic type $\left({ }^{\star} 1 \mathrm{a} /{ }^{\star} 15,{ }^{\star} 1 \mathrm{~b} / 15,{ }^{\star} 1 \mathrm{a} /{ }^{\star} 5\right)$, and weak metabolic type $\left({ }^{\star} 15 /{ }^{\star} 15\right.$, $\left.\star 5 /{ }^{\star} 5,{ }^{\star} /{ }^{\star} 15\right)$. The proportion of patients with three metabolic types in the Li people in Hainan Island is $75.5 \%$ for normal metabolic type, $21.7 \%$ for intermediate metabolic type and $2.78 \%$ for weak metabolic type. In the control group, $76.2 \%, 22.4 \%$ and $1.33 \%$ of the Han people in Hainan Island are normal, intermediate and weak metabolisms respectively. The $\chi^{2}$ test shows that there is no significant difference between two metabolic types in the Li people and the Han people in Hainan island except weak metabolic type $(\mathrm{P}<0.05)$ (Figure $2(\mathrm{~A}))$. 
Table 3. Distribution results of SLCO1B1 and ApoE gene polymorphisms of the Li people in Hainan.

\begin{tabular}{|c|c|c|c|c|c|c|c|c|c|}
\hline \multirow[t]{2}{*}{ Genotype } & & \multicolumn{2}{|c|}{$\begin{array}{l}\text { Protective } \\
\text { Genotype }\end{array}$} & \multicolumn{2}{|c|}{$\begin{array}{l}\text { Popular } \\
\text { Genotype }\end{array}$} & \multicolumn{2}{|c|}{$\begin{array}{c}\text { Risk } \\
\text { Genotype }\end{array}$} & \multirow{2}{*}{\multicolumn{2}{|c|}{$\begin{array}{c}{[\mathrm{n}(\%)]} \\
\text { Total }\end{array}$}} \\
\hline & & $\mathrm{e} 2 / \mathrm{e} 2$ & e2/e3 & $\mathrm{e} 2 / \mathrm{e} 4$ & e3/e3 & $\mathrm{e} 3 / \mathrm{e} 4$ & $\mathrm{e} 4 / \mathrm{e} 4$ & & \\
\hline \multirow{3}{*}{$\begin{array}{l}\text { Normal } \\
\text { Metabolic } \\
\text { Type }\end{array}$} & $1 \mathrm{a} / 1 \mathrm{a}$ & 0 & 4 & 1 & 21 & 5 & 3 & $34(6.77)$ & \multirow{3}{*}{$\begin{array}{c}379 \\
(75.5)\end{array}$} \\
\hline & $1 \mathrm{a} / 1 \mathrm{~b}$ & 0 & 19 & 3 & 95 & 19 & 0 & $136(27.09)$ & \\
\hline & $1 b / 1 b$ & 2 & 38 & 5 & 145 & 15 & 4 & $209(41.63)$ & \\
\hline \multirow{3}{*}{$\begin{array}{c}\text { Intermediate } \\
\text { Metabolic } \\
\text { Type }\end{array}$} & $1 \mathrm{a} / 5$ & 0 & 0 & 0 & 0 & 0 & 0 & $0(0)$ & \multirow{3}{*}{$\begin{array}{c}109 \\
(21.71)\end{array}$} \\
\hline & $1 \mathrm{a} / 15$ & 0 & 4 & 1 & 11 & 8 & 0 & $24(4.78)$ & \\
\hline & $1 b / 15$ & 0 & 19 & 2 & 49 & 15 & 0 & 85 (16.93) & \\
\hline \multirow{3}{*}{$\begin{array}{c}\text { Weak } \\
\text { Metabolic } \\
\text { Type }\end{array}$} & $5 / 5$ & 0 & 0 & 0 & 0 & 0 & 0 & $0(0)$ & \multirow{3}{*}{$\begin{array}{c}14 \\
(2.79)\end{array}$} \\
\hline & $5 / 15$ & 0 & 0 & 0 & 0 & 0 & 0 & $0(0)$ & \\
\hline & $15 / 15$ & 0 & 4 & 0 & 9 & 1 & 0 & $14(2.79)$ & \\
\hline$[\mathrm{n}(\%)]$ & & $2(0.4)$ & 89 (17.73) & $12(2.39)$ & $329(65.54)$ & $63(12.55)$ & 7 (1.39) & & \\
\hline Total & & \multicolumn{2}{|c|}{$91(18.13)$} & \multicolumn{2}{|c|}{$341(67.93)$} & \multicolumn{2}{|c|}{$70(13.94)$} & & \\
\hline
\end{tabular}

Table 4. Distribution results of SLCO1B1 and ApoE gene polymorphisms of the Han people in Hainan.

\begin{tabular}{|c|c|c|c|c|c|c|c|c|c|}
\hline \multirow[t]{2}{*}{ Genotype } & & \multicolumn{2}{|c|}{$\begin{array}{l}\text { Protective } \\
\text { Genotype }\end{array}$} & \multicolumn{2}{|c|}{$\begin{array}{l}\text { Popular } \\
\text { Genotype }\end{array}$} & \multicolumn{2}{|c|}{$\begin{array}{c}\text { Risk } \\
\text { Genotype }\end{array}$} & \multirow{2}{*}{\multicolumn{2}{|c|}{$\begin{array}{c}{[\mathrm{n}(\%)]} \\
\text { Total }\end{array}$}} \\
\hline & & $\mathrm{e} 2 / \mathrm{e} 2$ & $\mathrm{e} 2 / \mathrm{e} 3$ & $\mathrm{e} 2 / \mathrm{e} 4$ & e3/e3 & $\mathrm{e} 3 / \mathrm{e} 4$ & e4/e4 & & \\
\hline \multirow{3}{*}{$\begin{array}{c}\text { Normal } \\
\text { Metabolic } \\
\text { Type }\end{array}$} & $1 \mathrm{a} / 1 \mathrm{a}$ & 0 & 5 & 0 & 13 & 5 & 0 & $23(4.36)$ & \multirow{3}{*}{$\begin{array}{c}402 \\
(76.28)\end{array}$} \\
\hline & $1 \mathrm{a} / 1 \mathrm{~b}$ & 2 & 39 & 6 & 100 & 21 & 1 & $169(32.07)$ & \\
\hline & $1 b / 1 b$ & 1 & 38 & 3 & 143 & 25 & 0 & $210(39.85)$ & \\
\hline \multirow{3}{*}{$\begin{array}{c}\text { Intermediate } \\
\text { Metabolic } \\
\text { Type }\end{array}$} & $1 \mathrm{a} / 5$ & 0 & 0 & 0 & 0 & 0 & 0 & $0(0)$ & \multirow{3}{*}{$\begin{array}{c}118 \\
(22.39)\end{array}$} \\
\hline & $1 \mathrm{a} / 15$ & 0 & 5 & 1 & 22 & 4 & 0 & $32(6.07)$ & \\
\hline & $1 \mathrm{~b} / 15$ & 3 & 8 & 1 & 68 & 6 & 0 & $86(16.32)$ & \\
\hline \multirow{3}{*}{$\begin{array}{c}\text { Weak } \\
\text { Metabolic } \\
\text { Type }\end{array}$} & $5 / 5$ & 0 & 0 & 0 & 0 & 0 & 0 & $0(0)$ & \multirow{3}{*}{$\begin{array}{c}7 \\
(1.33)\end{array}$} \\
\hline & $5 / 15$ & 0 & 0 & 0 & 0 & 0 & 0 & $0(0)$ & \\
\hline & $15 / 15$ & 0 & 1 & 0 & 5 & 0 & 1 & $7(1.33)$ & \\
\hline [n (\%)] & & $6(1.14)$ & $6(18.22)$ & $11(2.09)$ & $351(66.60)$ & $61(11.57)$ & $2(0.38)$ & & \\
\hline Total & & \multicolumn{2}{|c|}{$102(19.35)$} & \multicolumn{2}{|c|}{$362(68.69)$} & \multicolumn{2}{|c|}{63 (11.95) } & & \\
\hline
\end{tabular}




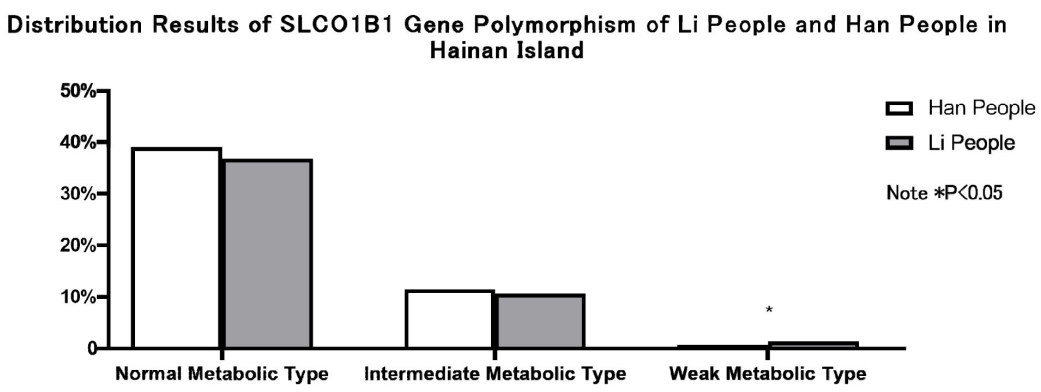

(A)

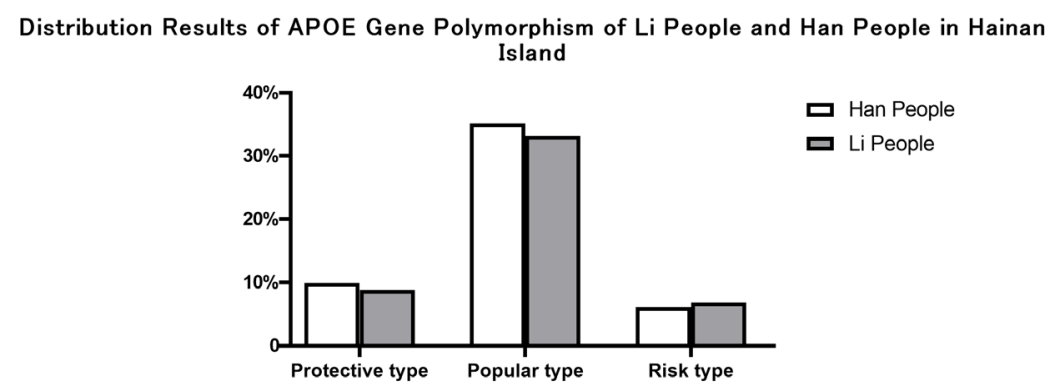

(B)

Figure 2. Distribution results of SLCO1B1 and ApoE gene polymorphism of the Li People and the Han People in Hainan Island. Note: (A) Distribution results of SLCO1B1 gene polymorphisms among different genders of the Li people in Hainan Island; (B) Distribution results of APOE gene polymorphisms among different genders of the Li People in Hainan Island.

\subsection{Analysis on Differences of the Proportions of Three Genotypes of APOE4 between the Li People and the Han People in Hainan Island}

The efficacy of statins is related with two loci of ApoE, namely ApoE4 388T>C, ApoE2 526C>T. According to the risk of cardiovascular disease and Alzheimer's disease caused by carrying different genotypes and the efficacy of statins, they can be divided into three categories: protective genotype (e2/e2, e2/e 3 are $\varepsilon 2$ phenotype); popular genotype (e2/e4, e3/e3 are $\varepsilon 3$ phenotype); risk genotype (e3/e4, e4/e4 are $\varepsilon 4$ phenotype). The results of this study show that the carrier rates of the above three genotypes in the $\mathrm{Li}$ people in Hainan Island are $18.12 \%$ for $\varepsilon 2,67.92 \%$ for $\varepsilon 3$ and $13.94 \%$ for $\varepsilon 4$. In the control group, the carrier rates of $\varepsilon 2$, $\varepsilon 3$ and $\varepsilon 4$ are $19.39 \%, 68.82 \%$ and $11.97 \%$ respectively. According to $\chi^{2}$ test, there is no significant difference in the above three genotypes between Li people and Han people in Hainan Island ( $\mathrm{P}>$ 0.05) (Figure 2(B)).

\subsection{Analysis of the Differences in the Distribution of SLC01B1 and ApoE Gene Polymorphisms among Different Genders of the Li People in Hainan Island}

This study analyzes two locus of SLCO1B1, namely SLCO1B1 ${ }^{\star} 1 \mathrm{~b}$ and SLCO1B1 ${ }^{\star} 5$ of the Li people in Hainan Island. The results show that the proportions of male genotypes are: ${ }^{\star} 1 \mathrm{a} /{ }^{\star} 1 \mathrm{a} 5.77 \%,{ }^{\star} 1 \mathrm{a} /{ }^{\star} 1 \mathrm{~b}$ $28.46 \%,{ }^{\star} 1 \mathrm{~b} / 1 \mathrm{~b} 41.92 \%,{ }^{\star} 1 \mathrm{a} /{ }^{\star} 50.00 \%,{ }^{\star} 1 \mathrm{a} /{ }^{\star} 153.85 \%,{ }^{\star} 1 \mathrm{~b} /{ }^{\star} 1517.31 \%,{ }^{\star} 5 /{ }^{\star} 50.00 \%,{ }^{\star} 5 /{ }^{\star} 150 \%,{ }^{\star} 15 /{ }^{\star} 15$ $2.79 \%$. The proportions of female genotypes are: ${ }^{\star} 1 \mathrm{a} /{ }^{\star} 1 \mathrm{a} 7.85 \%,{ }^{\star} 1 \mathrm{a} /{ }^{\star} 1 \mathrm{~b} 25.62 \%,{ }^{\star} 1 \mathrm{~b} / 1 \mathrm{~b} 41.32 \%,{ }^{\star} 1 \mathrm{a} /{ }^{\star} 5$ $0.00 \%,{ }^{\star} 1 \mathrm{a} /{ }^{\star} 155.79 \%,{ }^{\star} 1 \mathrm{~b} / 1516.53 \%,{ }^{\star} 5 /{ }^{\star} 50.00 \%,{ }^{\star} 5 /{ }^{\star} 150.00 \%,{ }^{\star} 15 /{ }^{\star} 152.89 \%$ (Figure $3(\mathrm{~A})$ ). The research of two locus of ApoE, namely ApoE2 and ApoE4 shows that the proportions of male genotypes are: e2/e2 $0.38 \%$, e2/e3 $18.46 \%$, e2/e4 $1.92 \%$, e3/e3 $65.00 \%$, e3/e4 $12.69 \%$, e4/e4 1.54\%; the proportions of female genotypes are: e2/e2 $0.41 \%$, e2/e3 $16.94 \%$, e2/e4 2.89\%, e3/e3 66.12\%, e3/e4 $12.40 \%$, e4/e4 $1.24 \%$ (Figure 3(B)). 


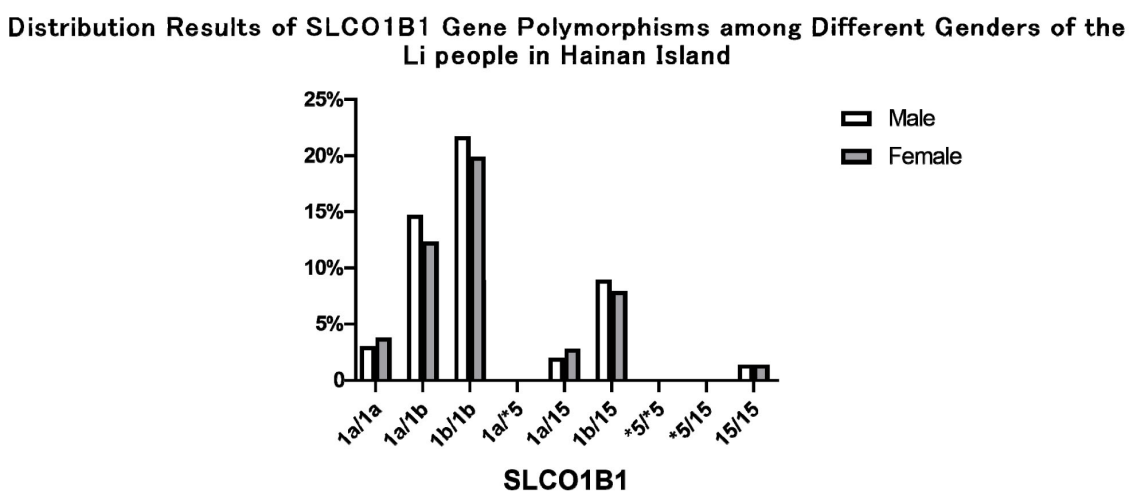

(A)

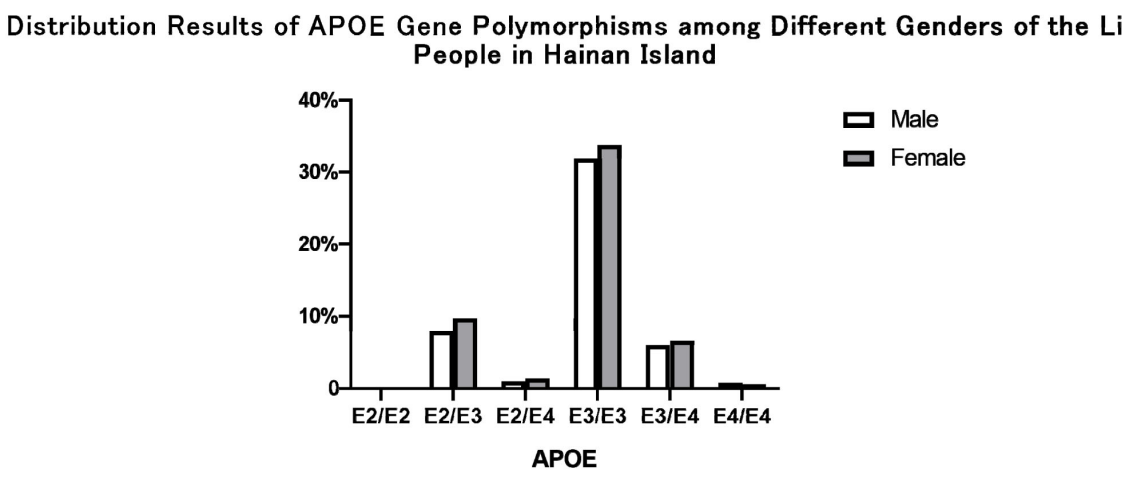

(B)

Figure 3. Distribution results of SLCO1B1 and ApoE gene polymorphisms among different genders of the Li people in Hainan Island. Note: (A) Distribution results of SLCO1B1 gene polymorphisms among different genders of the Li people in Hainan Island; (B) Distribution results of APOE gene polymorphisms among different genders of the Li People in Hainan Island.

The $\chi^{2}$ test shows that there is no significant difference in the distribution of SLCO1B1 and ApoE gene polymorphisms among different genders of the Li people in Hainan Island $(\mathrm{P}>0.05)$.

\subsection{Analysis of the Differences in the Distribution of SLCO1B1 and ApoE Gene Polymorphisms among Different Genders of the Han People in Hainan Island}

This study analyzes two locus of SLCO1B1, namely SLCO1B1* $1 \mathrm{~b}$ and SLCO1B1 ${ }^{\star} 5$ of the Han people in Hainan Island. The results show that the proportions of male genotypes are: ${ }^{\star} 1 \mathrm{a} /{ }^{\star} 1 \mathrm{a} 3.06 \%,{ }^{\star} 1 \mathrm{a} /{ }^{\star} 1 \mathrm{~b}$ $34.35 \%,{ }^{\star} 1 \mathrm{~b} / 1 \mathrm{~b} 40.82 \%,{ }^{\star} 1 \mathrm{a} /{ }^{*} 50.00 \%,{ }^{*} 1 \mathrm{a} /{ }^{\star} 153.74 \%,{ }^{*} 1 \mathrm{~b} /{ }^{\star} 1515.65 \%,{ }^{*} 5 /{ }^{\star} 50.00 \%,{ }^{\star} 5 /{ }^{\star} 150.00 \%,{ }^{*} 15 /{ }^{*} 15$ $2.38 \%$. The proportions of female genotypes are: ${ }^{\star} 1 \mathrm{a} /{ }^{\star} 1 \mathrm{a} 6.01 \%,{ }^{\star} 1 \mathrm{a} /{ }^{\star} 1 \mathrm{~b} 29.31 \%,{ }^{\star} 1 \mathrm{a} / 1 \mathrm{~b} 38.63 \%,{ }^{\star} 1 \mathrm{a} /{ }^{\star} 5$ $0.00 \%,{ }^{\star} 1 \mathrm{a} /{ }^{\star} 159.01 \%,{ }^{\star} 1 \mathrm{~b} / 1517.17 \%,{ }^{\star} 5 /{ }^{\star} 50.00 \%,{ }^{\star} 5 /{ }^{\star} 150.00 \%,{ }^{\star} 15 /{ }^{\star} 150.00 \%$ (Figure $4(\mathrm{~A})$ ). The $\chi^{2}$ test shows that there are significant differences in SLCO1B1 ${ }^{\star} 1 \mathrm{a} /{ }^{\star} 15$ and ${ }^{\star} 15 /{ }^{\star} 15$ between different genders of the Han people in Hainan Island $(\mathrm{P}<0.05)$, but no significant differences among the other genotypes $(\mathrm{P}>$ $0.05)$.

The research of two locus of ApoE, namely Apoe2 and ApoE4 shows that the proportions of male genotypes are: e2/e2 1.33\%, e2/e3 17.69\%, e2/e4 2.04\%, e3/e3 65.99\%, e3/e4 12.59\%, e4/e4 0.34\%; the proportions of female genotypes are: e2/e2 $0.86 \%$, e2/e3 18.53\%, e2/e4 2.16\%, e3/e3 67.17\%, e3/e4 10.34\%, e4/e4 $0.43 \%$ (Figure $4(\mathrm{~B})$ ). The $\chi^{2}$ test shows that there is no significant difference in the distribution of ApoE gene polymorphisms among different genders of the Han people in Hainan Island $(\mathrm{P}>0.05)$. 


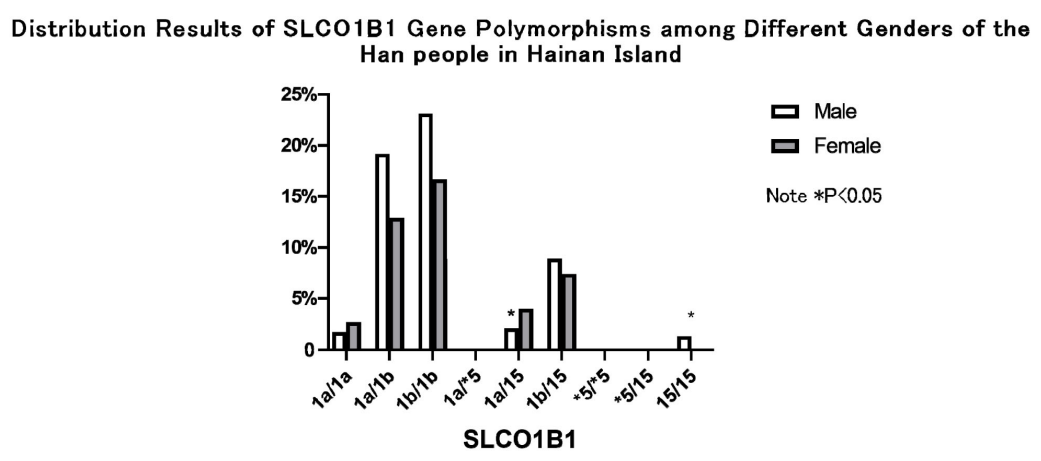

(A)

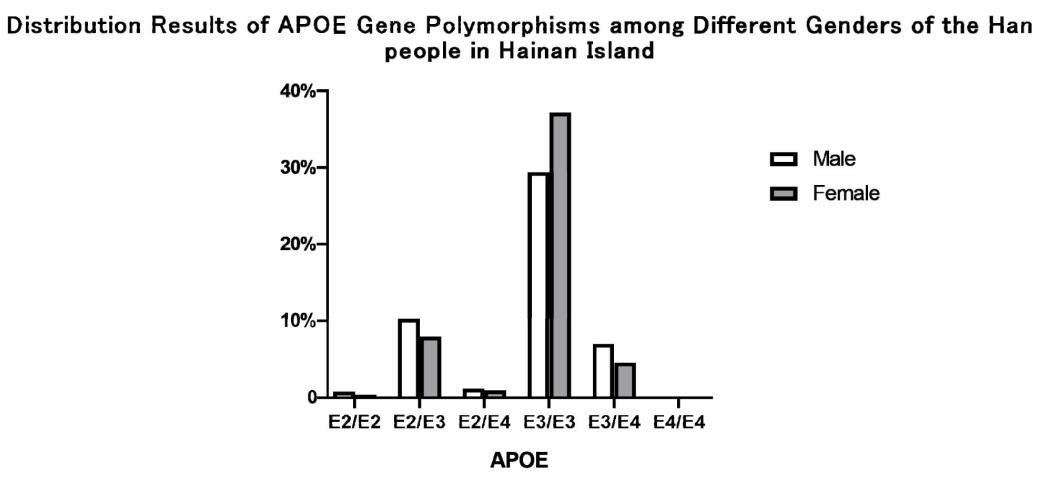

(B)

Figure 4. Distribution results of SLCO1B1 and ApoE gene polymorphisms among different genders of the Han people in Hainan Island. Note: (A) Distribution results of SLCO1B1 gene polymorphisms among different genders of the Han people in Hainan Island; (B) Distribution results of APOE gene polymorphisms among different genders of the Han people in Hainan Island.

\section{DISCUSSION}

With the rapid development of precision medicine and pharmacokinetics, the mode of clinical medication has changed from empirical medication to individualized medication. Studies have confirmed that SLCO1B1 and ApoE are two important genes that affect the pharmacokinetics and pharmacodynamics of statins, and the types of single nucleotide polymorphisms vary among different ethnic groups and regions [3].

According to Tang Bin [4] and other scholars, in Nanchang, China, the mutation rates of SLCO1B1 $388 \mathrm{~A}>\mathrm{G}$ and $521 \mathrm{~T}>\mathrm{C}$ in the Han people are $73.4 \%$ and $12.9 \%$, respectively. According to Tirona [5], for African Americans and Caucasian Americans, the mutation rates of SLCO1B1 388A $>\mathrm{G}$ and 521T $>\mathrm{C}$ are $74.0 \%, 30.0 \%$ and $2.0 \%, 14.0 \%$, respectively. Nishizato [6] and other scholars report that the mutation rates of Japanese population are $62.9 \%$ and $15.8 \%$. And according to Wang Jingwei [7] and other scholars, in Central China, the mutation rates of the Han people are $74.18 \%$ and $10.95 \%$. There are three common alleles $(\varepsilon 2, \varepsilon 3$, and $\varepsilon 4)$ of ApoE gene. The frequency range of $\varepsilon 2$ is $1 \%-15 \%$, that of $\varepsilon 3$ is $50 \%-90 \%$, and that of $\varepsilon 4$ is 5\% - 35\% [8]. According to literatures, the carrier rates of the Han people are $16.34 \%$ and $12.1 \%$ respectively $[7,9]$ in Central and South China. The above data fully show that SLCO1B1 and ApoE have high genetic heterogeneity, and the distribution of gene polymorphism has obvious differences among races and regions.

Hainan Island is the second largest island in China. According to the sixth census of Hainan Province in 2017, the total population of the Li minority group in Hainan Island is as high as 1, 490, 
013. Due to the customs of living together and non-intermarriage, the genotype composition of the Li people in Hainan Island is relatively single. So far, there is no literature report on the data of gene carrier rate of the Li people in Hainan Island. The results show that in Hainan Island, the mutation rates of SLCO1B1 $388 \mathrm{G}>\mathrm{A}$ and $521 \mathrm{~T}>\mathrm{C}$ are $77.29 \%$ and $13.65 \%$ respectively in the Li people, and $76.57 \%$ and $12.52 \%$ respectively in the Han people. The $\chi^{2}$ test indicates that there is no significant difference in the mutation rates of SLCO1B1 gene between the two nationalities $(\mathrm{P}>0.05)$. The mutation frequency of SLCO1B1 $388 \mathrm{G}>\mathrm{A}$ in Li people and Han people in Hainan Island is similar to that of the Han people in Nanchang and Central China and that of African Americans reported by Tang bin and Wang Jingwei [4, 7], but it is quite different from that of Caucasian Americans; While, the mutation frequency of SLCO1B1 521 T>C is similar to that of the Han people in Nanchang and Central China, and also similar to that of Japanese people and Caucasian Americans, but it is quite different from that of African Americans.

Therefore, obtaining the data of SLCO1B1 and ApoE gene polymorphism among different regions and different ethnic groups can guide clinicians to implement individualized medications based on patients' genotypes. And it also helps to further evaluate the effectiveness and safety of statin applications, which is the premise of precise treatment.

The most serious side effect of statins is myopathy. Studies have shown that mutant SLCO1B1 gene may lead to the decrease of the activity of encoded OATP1B1 transporter, which is manifested by the decreased ability of liver to absorb drugs, resulting in the increase of blood concentration of statins and increasing the risk of myopathy or rhabdomyolysis, endangering the life $[10,11]$. The results of this study show that in Hainan Island, the proportion of SLCO1B1 weak metabolic genotypes $\left({ }^{\star} 5 /{ }^{*} 5,{ }^{*} 5 /{ }^{*} 15,{ }^{\star} 15 /{ }^{\star} 15\right)$ in the Li people is $2.78 \%$, which was significantly higher than that in the Han people $(1.33 \%)$. It is also higher than the proportion in the Han people in Central China (1.31\%) reported by Wang Jingwei [7] and that in Northwest China (1.25\%) reported by Wang Haibin [12]. The above differences have statistical significance $(\mathrm{P}<0.05)$.

It can be seen that the proportion of SLCO1B1 weak metabolic genotypes of the Li people in Hainan Island is significantly higher than that of the Han people in China. For these patients, it is necessary to strictly control the dosage of the drug, monitor the adverse drug reaction to prevent the occurrence of high rhabdomyolysis or replace statins with other alternatives. In this study, a total of 14 cases of the Li people with SLCO1B1 weak metabolism genotype are detected, which indicates that this kind of people have poor ability to metabolize statins and have high risk of severe rhabdomyolysis. Therefore, it is not suitable to use statin lipid-lowering drugs. It is recommended to use ezetimibe, probucol or other lipid-lowering drugs in clinical treatment.

The protein encoded by the ApoE gene is plasma protein, which is involved in lipid metabolism and the transport of cholesterol and triglycerides, located on chromosome 19q13.2. It has two nonsynonymous polymorphisms (rs429358 and rs7412), and can form 3 common alleles ( $22, \varepsilon 3, \varepsilon 4)$. According to Wei Faquan [13] and other scholars, through the Meta-analysis of the relationship between the gene polymorphism of apolipoprotein E and the efficacy of statins in the Chinese population, they find that ApoE gene polymorphism is related to the efficacy of statins. For $\varepsilon 2$ allele carriers, statins have better efficacy of lipid-lowering, but for $\varepsilon 4$ allele carriers the efficacy is just the opposite. In recent years, more and more studies have shown that ApoE $\varepsilon 4$ carriers are at higher risk of coronary heart disease, senile dementia, cerebral infarction, and diabetes, and that statins have poor efficacy of lipid-lowering [14-19]. Some literatures indicates that for ApoEe4 carriers, the risk of Alzheimer's disease is 4 times higher than that of other populations, and the age of onset is nearly 20 years earlier [20]. People with e3/e4 heterozygous type have potential to get Alzheimer's disease, while those with e4/e4 homozygous type have more potential [21]. For such people with hyperlipidemia, to achieve better lipid-lowering effects, low-dose statins should be used in combination with other lipid-lowering drugs. The results of this study show that in Hainan Island, the frequency range of ApoE gene $\varepsilon 4$ of the Li people is $13.94 \%$, and in the control group, that of the Han people is $11.97 \%$. The $\chi^{2}$ test shows that the difference between Li people and Han people in Hainan Island 
has no statistical significance $(\mathrm{P}>0.05)$. The $\varepsilon 4$ risk gene carrier rate of Li people and Han people in Hainan Island is close to that of the Han people in South China (12.1\%) and Central China (16.31\%) reported by Wang Jingwei [7] and other scholars. This suggests that there is no significant difference in the frequency of ApoE gene $\varepsilon 4$ among them.

This study finds that the e4/e4 homozygous carrier rate of the Li people in Hainan Island is $1.39 \%$. According to foreign scholars such as Stefany [22], the e4/e4 homozygous carrier rates of Caucasian Americans, African Americans, Hispanics, and Japanese are 1.8\%, 2.1\%, 1.9\%, 0.8\%. This data shows that the e4/e4 homozygous carrier rate of the Li people in Hainan Island is higher than that of the Japanese, and lower than that of Caucasian Americans, African Americans and Hispanics. The immediate family members of e4/e4 homozygous carriers should undergo ApoE genetic testing and genetic counseling, which is of great significance for patients' health and rational drug use.

\section{CONCLUSION}

In conclusion, according to the detailed data obtained from the analysis of SLCO1B1 and apoE gene polymorphisms of the $\mathrm{Li}$ people in Hainan Island, it can provide guidance for individual medication of the Li people in Hainan Island, and further improve the safety and efficacy of statins. And it also provides experimental data support for the prevention of coronary heart disease, cardiovascular and cerebrovascular diseases, Alzheimer's disease and other diseases of the Li people in Hainan Island, so as to achieve the goal of predicting the risk of cardiovascular and cerebrovascular diseases in advance and offering precise treatment for each patient.

\section{ACKNOWLEDGEMENTS}

This study was supported by the Medical and Health Research Projects of Hainan Province (2001320119A2001).

\section{CONFLICTS OF INTEREST}

The authors declare no conflicts of interest regarding the publication of this paper.

\section{REFERENCES}

1. Xiao, Q.L., Wang, Y.Q., et al. (2016) Adverse Reactions and Rational Clinical Application. Journal of Clinical Rational Use of Statins, $9,78$.

2. Superko, H.R., Momary, K.M. and Li, Y. ( 2012) Statins Personalized. Medical Clinics of North America, 96, 123-139. https://doi.org/10.1016/j.mcna.2011.11.004

3. Guan, Z.W., Wu, K.R., Li, R., Yin, Y., Li, X.L., Zhang, S.F. and Li, Y. (2019) Pharmacogenetics of Statins Treatment: Efficacy and Safety. Journal of Clinical Pharmacy and Therapeutics, 44, 858-867. https://doi.org/10.1111/jcpt.13025

4. Tang, B., Huang, D., Fu, Q., et al. (2014) The Distribution of SLCO1B1 Gene in the Han People with Primary Hyperlipidemia in Nanchang Area. Modern Chinese Doctor, 52, 1-3.

5. Tirona, R.G., Leake, B.F., Merino, G. and Kim, R.B. (2001) Polymorphisms in OATP-C: Identification of Multiple Allelic Variants Associated with Altered Transport Activity among European- and African-Americans. The Journal of Biological Chemistry, 276, 35669-35675. https://doi.org/10.1074/jbc.M103792200

6. Nishizato, Y., Ieiri, I., Suzuki, H., Kimura, M., Kawabata, K., Hirota, T., Takane, H., Irie, S., Kusuhara, H., Urasaki, Y., Urae, A., Higuchi, S., Otsubo, K. and Sugiyama, Y. (2003) Polymorphisms of OATP-C (SLC21A6) and OAT3 (SLC22A8) Genes: Consequences for Pravastatin Pharmacokinetics. Clinical Pharmacology and Therapeutics, 73, 554-565. https://doi.org/10.1016/S0009-9236(03)00060-2 
7. Wang, J.W., Li, Y., Qiao, B., Chen, J.J., et al. (2018) The Polymorphisms of SLCO1B1 and ApoE in the Han People in Central China. Journal of Practical Medicine, 34, 3041-3046.

8. Mahley, R.W. and Rall, S.C. (2000) Apolipoprotein E: Far More than a Lipid Transport Protein. Annual Review of Genomics and Human Genetics, 1, 507-537. https://doi.org/10.1146/annurev.genom.1.1.507

9. Li, X., Xu, Y.J., Luo, E., et al. (2017) The Analysis of SLCO1B1 and ApoE Gene Polymorphisms in the Han People in South China. Laboratory Medicine and Clinics, 6, 773-775.

10. Bakar, N.S., Neely, D., Avery, P., et al. (2018) Genetic and Clinical Factors Are Associated with Statin-Related Myotoxicity of Moderate Severity: A Case-Control Study. Clinical Pharmacology \& Therapeutics, 104, 178-187. https://doi.org/10.1002/cpt.887

11. Hou, Q., Li, S., Li, L., Li, Y., Sun, X. and Tian, H. (2015) Association between SLCO1B1 Gene T521C Polymorphism and Statin-Related Myopathy Risk: A Meta-Analysis of Case-Control Studies. Medicine, 94, e1268. https://doi.org/10.1097/MD.0000000000001268

12. Wang, H.B., Zhang, D.Q., Zhao, J., Si, Y.L., et al. (2019) The Analysis of SLCO1B1 and ApoE Gene Polymorphisms in 2633 Elderly Patients in the Han People. Journal of Chinese PLA Medical College, 40, 887-891.

13. Wei, F.Q., Wang, S., et al. (2015) The Meta-Analysis of the Relationship between the Polymorphisms of Apolipoprotein E Gene and the Efficacy of Statins in Chinese Population. Clinical Meta-Analysis, 30, 486-491.

14. Ostrowitzki, S., et al. (2017) A Phase III Randomized Trial of Gantenerumab in Prodromal Alzheimer's Disease. Alzheimer's Research \& Therapy, 9, 95.

15. Jiang, Y., Ma, L., Han, C., Liu, Q., Cong, X., Xu, Y., Zhao, T., Li, P. and Cao, Y. (2017) Effects of Apolipoprotein E Isoforms in Diabetic Nephropathy of Chinese Type 2 Diabetic Patients. Journal of Diabetes Research, 2017, Article ID: 3560920. https://doi.org/10.1155/2017/3560920

16. Liu, W.X., Yang, L.P., et al. (2016) Study on the Correlation between Type 2 Diabetes and the Polymorphism of Apolipoprotein Gene in Naxi Nationality in Yunnan Province. Chinese Journal of Diabetes, 24, 402-406.

17. Zhang, J., Zhou, X.H., et al. (2016) Mild Cognitive Dysfunction and Apolipoprotein E in Chinese Population. Journal of Clinical Medicine, 31, 402-406.

18. Wu, P.L., Zhao, M.S., et al. (2019) Research Progress in Alzheimer's Disease and Age-Related Cataracts. International Journal of Geriatrics, 40, 40-50.

19. Huang, J.Z., Wu, Q., Chen, D.P., et al. (2019) Correlation between Early-Onset Coronary Heart Disease, Non-Early-Onset Coronary Heart Disease and the Polymorphisms of Apolipoprotein E Gene. Lingnan Journal of Emergency Medicine, 24, 141-143.

20. Liu, C.-C., Kanekiyo, T., Xu, H.X., et al. (2013) Apolipoprotein E and Alzheimer Disease: Risk, Mechanisms, and Therapy. Nature Reviews Neurology, 9, 106-118. https://doi.org/10.1038/nrneurol.2012.263

21. Lutz, M.W., Crenshaw, D., Welsh-Bohmer, K.A., Burns, D.K. and Roses, A.D. (2016) New Genetic Approaches to AD: Lessons from APOE-TOMM40 Phylogenetics. Current Neurology and Neuroscience Reports, 16, 48. https://doi.org/10.1007/s11910-016-0643-8

22. Montufar, S., Calero, C., et al. (2017) Association between the APOE 4 Allele and Late-Onset Alzheimer's Disease in an Ecuadorian Mestizo Population. International Journal of Alzheimer's Disease, 2017, Article ID: 1059678. https://doi.org/10.1155/2017/1059678 